\title{
Morphological polymorphism of Trypanosoma copemani and description of the genetically diverse $T$. vegrandis sp. nov. from the critically endangered Australian potoroid, the brush-tailed bettong (Bettongia penicillata (Gray, 1837))
}

Craig K Thompson ${ }^{1 *}$, Adriana Botero ${ }^{1}$, Adrian F Wayne ${ }^{2}$, Stephanie S Godfrey ${ }^{1}$, Alan J Lymbery ${ }^{3}$ and RC Andrew Thompson' ${ }^{1}$

\begin{abstract}
Background: The trypanosome diversity of the Brush-tailed Bettong (Bettongia penicillata), known locally as the woylie, has been further investigated. At a species level, woylies are critically endangered and have declined by $90 \%$ since 1999 . The predation of individuals made more vulnerable by disease is thought to be the primary cause of this decline, but remains to be proven.

Methods: Woylies were sampled from three locations in southern Western Australia. Blood samples were collected and analysed using fluorescence in situ hybridization, conventional staining techniques and microscopy. Molecular techniques were also used to confirm morphological observations.

Results: The trypanosomes in the blood of woylies were grouped into three morphologically distinct trypomastigote forms, encompassing two separate species. The larger of the two species, Trypanosoma copemani exhibited polymorphic trypomastigote forms, with morphological phenotypes being distinguishable, primarily by the distance between the kinetoplast and nucleus. The second trypanosome species was only $20 \%$ of the length of T. copemani and is believed to be one of the smallest recorded trypanosome species from mammals. No morphological polymorphism was identified for this genetically diverse second species. We described the trypomastigote morphology of this new, smaller species from the peripheral blood of the woylie and proposed the name T. vegrandis $\mathrm{sp}$. nov. Temporal results indicate that during T. copemani Phenotype 1 infections, the blood forms remain numerous and are continuously detectable by molecular methodology. In contrast, the trypomastigote forms of T. copemani Phenotype 2 appear to decrease in prevalence in the blood to below molecular detectable levels.
\end{abstract}

Conclusions: Here we report for the first time on the morphological diversity of trypanosomes infecting the woylie and provide the first visual evidence of a mixed infection of both $T$. vegrandis sp. nov and T. copemani. We also provide supporting evidence that over time, the intracellular T. copemani Phenotype 2 may become localised in the tissues of woylies as the infection progresses from the active acute to chronic phase. As evidence grows, further research will be necessary to investigate whether the morphologically diverse trypanosomes of woylies have impacted on the health of their hosts during recent population declines.

Keywords: Trypanosoma vegrandis, T. copemani, Morphology, PCR, Sequencing, Fluorescence, Woylie, Bettongia, penicillata

\footnotetext{
* Correspondence: C.Thompson@murdoch.edu.au

${ }^{1}$ School of Veterinary and Life Sciences, Murdoch University, South Street,

Western Australia 6150, Australia

Full list of author information is available at the end of the article
} 


\section{Background}

Trypanosomes are parasitic protozoans (Sarcomastigophora: Kinetoplastida), which cause disease and death in humans and livestock around the world. Trypanosoma cruzi (Chagas disease) and T. brucei (African sleeping sickness) are collectively responsible for about 63,000 human deaths per year [1], while T. evansi (Surra), $T$. vivax (Nagana) and T. congolense (Nagana) are all of great economic concern to livestock production in Africa, Asia and South America [2-4].

By contrast, the trypanosomes of wildlife have been poorly studied. There is however some evidence indicating that these parasitic protozoans may be the causative agents behind population declines and extinctions of endangered fauna. For example, it was 100 years after a report of the extinction of endemic native rats on Christmas Island before evidence was presented identifying $T$. lewisi as possibly being influential during the disappearance of the native rats $[5,6]$. Recent studies have shown that the unintentional introduction of the black rat (Rattus rattus) and its fleas infected with $T$. lewisi may have contributed to the extinction of $R$. macleari and possibly $R$. nativitatis [5,7].

It is also possible that trypanosomes have played a potential role during the recent decline of the woylie (Bettongia penicillata) in Western Australia (WA) [8,9]. Prior to European settlement of Australia in 1788, woylies were distributed over much of the southern half of mainland Australia [10]. However, as a consequence of human expansion and introduced exotic predators, the natural abundance and distribution of the woylie has been severely challenged [10-12]. By the 1970s woylies became restricted to four small populations located in south-western Australia only; namely Tutanning Nature Reserve, Dryandra Woodlands and the Upper Warren Region (UWR) (which includes the Perup and Kingston populations) [11,13] (Figure 1).
The systematic, broadscale control of foxes using 1080-poison began in the 1970's and by 1996 the woylie became the first Australian taxon to have its conservation status downgraded from "Endangered" to "Low Risk / Conservation Dependent" (IUCN Red List) because of recovery efforts $[11,12]$. However, despite the continuous effort to control exotic predators, woylie populations have undergone a further and rapid decline since 1999 [14-16]. The factors responsible for this recent and rapid decline remain unclear, with the future survival of indigenous woylies becoming increasingly uncertain. However, recent spatio-temporal population modelling has hypothesised that disease, in conjunction with predation, may have been the main contributing factors to the recent woylie population declines [17].

During a recent disease investigation, trypanosomes were identified in the blood of woylies from the UWR [9]. The trypanosomes identified were a morphologically distinct species endemic to Australia, which infected between $35 \%$ (by molecular techniques) and 43\% (by microscopy) of woylies in these declining populations [9]. In an effort to further investigate the influence of trypanosomes upon the recent decline, research was extended to include woylies at the Karakamia Wildlife Sanctuary (KWS) (Figure 1), as this 285 hectare feralfree enclosure contained the only stable high-density sub-population of woylies on mainland Australia [18]. Molecular techniques identified a trypanosome prevalence of $14 \%$ at KWS, with microscopy failing to identify any morphological forms in the blood [9]. The observed higher parasite prevalence and parasitaemia (as interpreted by microscopy) in the declining Upper Warren populations helped strengthen the potential role of disease in the recent woylie decline [9].

The present study extends previous work and examines the morphology of the trypanosomes infecting woylies at both the UWR (with eight woylies translocated to NAR as

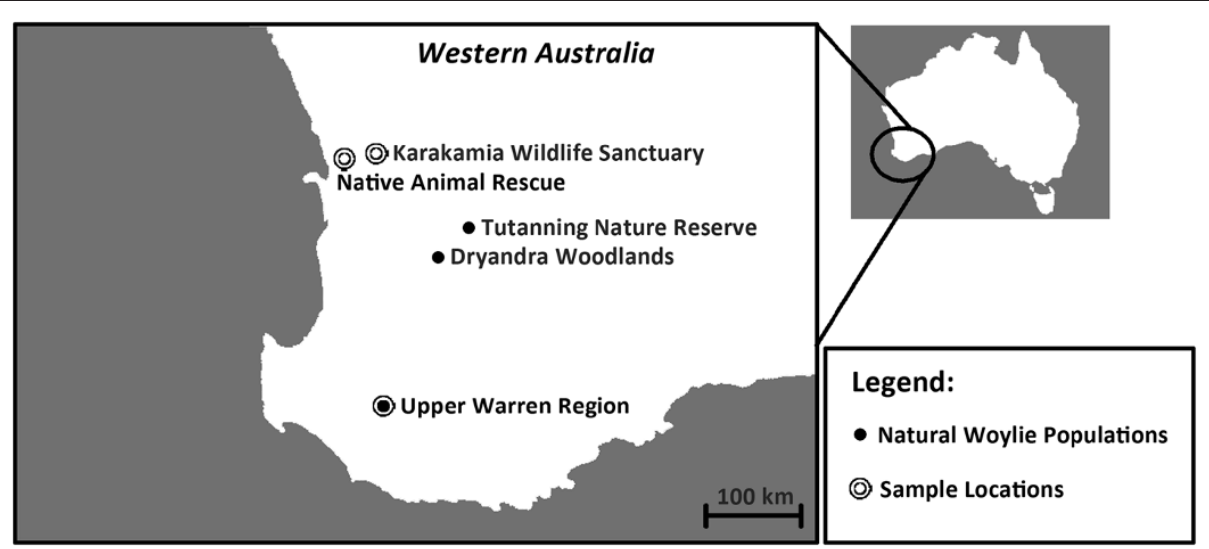

Figure 1 Remaining natural populations of woylies and the sample locations for this study in Western Australia. 
part of a more intensive observational study) and at KWS. We report for the first time on two different morphological phenotypes of $T$. copemani infecting woylies. We also report the first visual identification of a second smaller trypanosome, which is believed to be one of the smallest recorded trypanosomes from mammals. The morphology of this new species has been described and named T. vegrandis sp. nov. Also presented is the first visual identification of a mixed infection of both $T$. copemani and $T$. vegrandis sp. nov.

\section{Methods}

\section{Sample collection and preparation}

Sheffield traps baited with a mixture of rolled oats, peanut butter and sardines were used to capture woylies from three separate locations in WA. Firstly from the UWR (Figure 1) during November and December, 2010. UWR is predominantly publicly-owned conservation estate and state forest, managed by the Department of Environment and Conservation (DEC), and supports the largest wild woylie population and two of the four indigenous genetically distinct subpopulations extant at the time [13]. Secondly at Native Animal Rescue (NAR) (Figure 1) on seven separate occasions between April 2011 and April 2012. NAR is managed by the Fauna Rehabilitation Foundation, where a predator proof enclosure $(110 \mathrm{~m}$ x $70 \mathrm{~m})$ has been purpose-built to house 16 woylies. Thirdly at KWS (Figure 1) on two separate occasions during September 2011 and February 2012. KWS is managed by Australian Wildlife Conservancy, where a predator proof fence has been constructed for native Western Australian endangered mammals.

After removing the animal from the trap, a $400 \mu \mathrm{l} \mathrm{sam-}$ ple of blood was collected from the lateral caudal vein using a $25 \mathrm{G}$ x 5/8" needle and $1 \mathrm{ml}$ syringe. From the collected blood, $300 \mu \mathrm{l}$ was placed into a MiniCollect $1 \mathrm{ml}$ EDTA tube (Greiner bio-one, Germany) to prevent clotting and kept at $4^{\circ} \mathrm{C}$ for DNA extraction and PCR. With the remaining blood, multiple thin blood smears were made from each woylie sampled. Wet mounted slides were also collected during the February 2012 sampling at KWS. After blood collection, woylies were released at the point of capture, except for eight woylies from the UWR, which were translocated to NAR for release.

\section{Fluorescence in situ hybridisation (FISH) and staining}

Using Trypanosoma Clade B internal forward primer sequence (TVIF [5'- GAC CAA AAA CGT GCA CGT G -3']) [19], a commercially synthesised probe was manufactured which bound an AlexaFluor350 label at the $5^{\prime}$ end (BioSynthesis, Texas, USA). The AlexaFluro350 label excites at $350 \mathrm{~nm}$ and emits at $442 \mathrm{~nm}$.

The FISH protocol used in this study was modified from that developed by $\mathrm{Li}$ [20] and was conducted within 24 hours of blood collection. After application of a $125 \mu \mathrm{l}$ Frame-Seal Incubation Chamber (Bio-Rad, California, USA) to the dried blood slide, cells within the chamber were fixed with $120 \mu \mathrm{l}$ of $4^{\circ} \mathrm{C}$ fixative buffer $\left(88 \mu \mathrm{l}\right.$ of $95 \%$ ethanol, $20 \mu \mathrm{l}$ of deionised $\mathrm{H}_{2} \mathrm{O}$ and $12 \mu \mathrm{l}$ of $25 \mathrm{x}$ SET buffer [3.75 M NaCl, $25 \mathrm{mM}$ EDTA, $0.5 \mathrm{M}$ Tris $\mathrm{HCl} @ \mathrm{pH} 7.8])$. The buffer was left to incubate at $4^{\circ} \mathrm{C}$ for 40 minutes. The fixative buffer was then drained from the chamber using filter paper and washed with PBS $\left(950 \mathrm{ml}\right.$ of distilled $\mathrm{H}_{2} \mathrm{O}$, and $50 \mathrm{ml}$ of 20x PBS stock solution [160.0 g/L of NaCl, $24.2 \mathrm{~g} / \mathrm{L}$ of $\mathrm{KH}_{2} \mathrm{PO} 4$ and $6.8 \mathrm{~g} / \mathrm{L}$ of $\left.\mathrm{K}_{2} \mathrm{HPO}_{4}\right]$ ). After drying the slide in an incubation oven at $58^{\circ} \mathrm{C}$ for 15 minutes, the cells within the chamber were dehydrated using 50\%, $80 \%$ and $96 \%$ ethanol steps for a period of 90 seconds each, after which, the slide was allowed to air dry.

The remaining steps of the FISH protocol were conducted in a darkened room. To the slide chamber, $125 \mu$ l of hybridisation mix $(2 \mu$ l of TVIF probe $(20 \mu \mathrm{M})$, 5x SET buffer, Igepal-CA630 [Sigma, Castle Hill, NSW, Australia] and $25 \mu \mathrm{g} / \mathrm{ml}$ polyA potassium salt [Sigma, Castle Hill, NSW, Australia]) was added and the slide placed into an incubation oven at $58^{\circ} \mathrm{C}$ for 90 minutes. The hybridisation mix was removed from the chamber with filter paper and the cells within the chamber were washed with $1 \mathrm{x}$ SET buffer preheated to $58^{\circ} \mathrm{C}$. An additional $125 \mu \mathrm{l}$ of the preheated 1x SET buffer was added to the slide chamber and the slide placed into an incubation oven at $58^{\circ} \mathrm{C}$ for 15 minutes; this step was repeated once more. The $1 \mathrm{x}$ SET buffer was drained out of the chamber with filter paper and the frame-seal removed. The slide was allowed to air dry in the darkened room.

Once dried, the slide was then stained with Modified Wright's stain in the darkened room and allowed to dry again. Due to an auto-fluorescence issue with anti-fading agents and the Modified Wright's stain, a drop of distilled water was placed onto the slide and covered with a $50 \mathrm{~mm}$ cover slip. The edges of the cover slip were sealed with clear nail-polish to prevent evaporation. The slide was then placed into a dark storage box until viewed under the microscope.

\section{Microscopy and image acquisition}

The hybridised and stained slides were examined with a BX51 microscope (Olympus, Japan) using white light, as well as ultraviolet light $(330-385 \mathrm{~nm})$ through an emission filter $(420 \mathrm{~nm})$. The Alexafluor350 probe fluoresced bright blue under the ultraviolet light conditions. Slides were scanned using the $40 \mathrm{x}$ objective lens, with digital images captured using the $100 x$ objective lens. Digital images with an inserted scale bar were captured in a TIFF file format using a microscope mounted camera and DP Controller (Olympus, Japan). Measurements of the key morphological features as described by Hoare 
[21] were made using Adobe Photoshop CS5 Extended (Adobe Systems Incorporated, USA).

\section{Morphology}

Key morphological measurements were recorded for each of the trypanosomes observed. Morphological traits recorded included total length (L) (including free flagellum), width over the nucleus (W), distance of the posterior to kinetoplast (PK), posterior to nucleus (PN), kinetoplast to nucleus $(\mathrm{KN})$, anterior to nucleus (NA) and length of the free flagellum (FF). For T. copemani the kinetoplast-length (K-l) and kinetoplast-width (K-w) were also measured.

Two additional ratios were calculated as they have been used previously to discriminate species of trypanosomes [21]; the Nucleus Index (NI) $(=\mathrm{PN} / \mathrm{NA})$ and the Kinetoplast Index $(\mathrm{KI})(=\mathrm{PN} / \mathrm{KN})$. When $\mathrm{NI}=1$, the nucleus is in the middle of the body; $\mathrm{NI}<1$, the nucleus is posteriorly located in the body; and N1>1, the nucleus is anteriorly located in the body [21]. When $\mathrm{KI}=2$, the kinetoplast is half way between the posterior and nucleus; $\mathrm{KI}<2$, the kinetoplast is closer to the posterior than to the nucleus; and $\mathrm{KI}>2$, the kinetoplast is closer to the nucleus than the posterior end of the body [21].

Differences in morphology between groups of trypanosomes were tested over all morphological traits (except the two ratio traits) with multivariate analysis of variance (MANOVA), and differences between groups for each trait (including the ratio traits) were tested by single factor analyses of variance, using a Bonferroni correction to maintain an experiment-wide Type I error rate of $5 \%$. If the MANOVA showed a significant difference between groups, then discriminant analysis was used to detect the best combination of traits separating the groups. All statistical analyses were conducted with the software JMP 10.0 [22].

\section{DNA Extraction}

Blood collected in EDTA tubes were used for genomic DNA extraction. DNA was extracted from $300 \mu$ l of host blood using the Wizard Genomic DNA Purification Kit (Cat\# A1125) as per the protocol for whole blood extraction (Promega, Wisconsin USA). DNA was eluted in $60 \mu \mathrm{l}$ of DNA Rehydration Solution and stored at $-20^{\circ} \mathrm{C}$ prior to use. A negative control was included in each batch of DNA extractions, which contained no blood.

\section{Clade-specific PCR}

Three separate clade-specific nested PCR protocols were used to amplify the trypanosome $18 \mathrm{~S}$ rDNA region using primers and PCR reactions as previously described $[19,23]$. However, this study used different PCR conditions for four of the primer pairs. For T. copemani external primers (S825F and SLIR) [19], the pre-PCR step was
1 cycle of $94^{\circ} \mathrm{C}$ for 5 mins, $50^{\circ} \mathrm{C}$ for 2 mins and $72^{\circ} \mathrm{C}$ for 4 mins, followed by 35 cycles of $94^{\circ} \mathrm{C}$ for 30 secs, an annealing temperature of $57^{\circ} \mathrm{C}$ for 30 secs and an extension temperature of $72^{\circ} \mathrm{C}$ for 2 mins 20 secs, with a final step of $72^{\circ} \mathrm{C}$ for 7 mins. For T. copemani internal primers (WoF and WoR) [23] and Clade B external and internal primers (TVEF, TVER, TVIF, TVIR) [19], the annealing temperature for the 35 cycles was $58^{\circ} \mathrm{C}$ for 30 secs, while the extension temperature was the same but held for only 50 seconds per cycle. T. gilletti species-specific primer sets were used as previously described [23].

Four controls were used in every nested PCR and included the negative control from the DNA extraction, a primary and a secondary PCR negative control and PCR positive control. All were monitored to ensure reliability of results. PCR products were run on a $1.5 \%$ agarose gel using SYBR Safe Gel Stain (Invitrogen, California USA) and visualized by illumination with UV light.

\section{Sequencing PCR}

A fourth nested PCR was used to amplify positive samples of T. copemani and T. vegrandis sp. nov. for sequencing. This technique targeted the $18 \mathrm{~S}$ rDNA region and used the primers and PCR reaction as previously described [19] but with different PCR conditions for each of the three primer pairs. For the external primers SLF and S762R, the pre-PCR step was 1 cycle of $94^{\circ} \mathrm{C}$ for 5 mins, $50^{\circ} \mathrm{C}$ for 2 mins and $72^{\circ} \mathrm{C}$ for 4 mins, followed by 35 cycles of $94^{\circ} \mathrm{C}$ for 30 secs, an annealing temperature of $55^{\circ} \mathrm{C}$ for 30 secs and $72^{\circ} \mathrm{C}$ for 2 mins 20 secs, with a final step of $72^{\circ} \mathrm{C}$ for 7 mins. For the first pair of internal primers S823F and S662R, the annealing temperature for the 35 cycles was $56^{\circ} \mathrm{C}$ for 30 secs, while for the second pair of internal primers S825F and SLIR, the annealing temperature for the 35 cycles was $57^{\circ} \mathrm{C}$ for 30 secs. The combination of these two PCR products amplified a $1410 \mathrm{bp}$ amplicon for sequencing. Four controls were used as described above. PCR products were run on a 1.5\% agarose gel using SYBR Safe Gel Stain (Invitrogen, California USA) and visualized by illumination with UV light.

PCR products of appropriate size were purified using the Agencourt AMPure PCR Purification system (Beckman Coulter, California USA) as per the manufacturer's instructions and sequenced using an ABI Prism ${ }^{\mathrm{TM}}$ Terminator Cycle Sequencing Kit (Applied Bio-systems, California USA) on an Applied Bio-System 3730 DNA Analyser. The resulting sequences were then analysed and aligned using ClustalX 2.1.

\section{Results}

\section{Trypanosoma copemani- microscopy and image acquisition}

During the trapping sessions at the UWR, eight of the 15 blood samples examined by microscopy were identified positive for T. copemani infections. From these eight 
infected woylies, a total of 110 trypanosomes were identified in the blood smears. All were trypomastigote forms and their overall measurements are presented in Table 1. The trypomastigotes observed were both broad $(\mathrm{N}=78)$ (Figure 2A) and slender $(\mathrm{N}=32)$ (Figure 2B). No divisional stages of the trypanosomes were observed in the blood smears. These same eight positive woylies were translocated to the purpose built enclosure at NAR and were part of the temporal study undertaken there.

When considering the phylogenetic analysis presented by Botero et al., [19] and the sequencing results below, the 110 trypomastigotes measured were separated into two groups. The first group was "T. copemani Phenotype 1" (P1) comprising trypomastigotes found in Woylie ID: WC2741, WC2830, WC2842, WC2844 \& WC2920 and the second was "T. copemani Phenotype 2" (P2) comprising trypomastigotes found in Woylie ID: WC2807, WC2841 \& WC2930. Mean morphological traits for T. copemani P1 (Figure 3A) and T. copemani P2 (Figure 3B) are shown in Table 1.

From the MANOVA there was a significant morphological difference between groups $\left(F_{9,100}=22.06\right.$, $\mathrm{P}<0.0001)$. Univariate tests found significant differences between groups in $\mathrm{KN}\left(\mathrm{F}_{1,108}=131.42\right), \mathrm{L}\left(\mathrm{F}_{1,108}=34.10\right)$, KI $\left(\mathrm{F}_{1,108}=22.73\right), \mathrm{K}-\mathrm{l}\left(\mathrm{F}_{1,108}=13.98\right), \mathrm{PN}\left(\mathrm{F}_{1,108}=12.11\right)$ and NA $\left(\mathrm{F}_{1,108}=10.60\right)(\mathrm{P}<0.05$ for all tests, with the Bonferroni). Discriminant analysis correctly classified 95.5\% of the original grouped cases along one canonical discriminant function, which loaded most heavily for $\mathrm{KN}$ (0.78), L (0.40), K-1 (-0.26), PN (0.24) and NA (0.22).

\section{Trypanosoma copemani- PCR and sequencing}

The clade-specific PCR confirmed the presence of T. copemani in the eight woylies at the time of translocation from the UWR to NAR. Two distinct genotypes were also identified by sequencing, having a 13 base-pair variation over the larger combined $1410 \mathrm{bp}$ amplicon. The grouping of the eight infected woylies based on the two different genotypes was the same as the phenotypic grouping presented above.

The temporal molecular analysis of infected woylies at NAR between April 2010 and April 2011 indicated that the five woylies infected with $T$. copemani P1 remained PCR positive throughout the study period (Table 2). The November 2011 and April 2012 positive samples were sequenced and confirmed the continued presence of T. copemani P1. On the one and only occasion PCR failed to identify the presence of $T$. copemani in these five woylies (WC2842 during Dec 2011; < $50 \mu$ l of blood was collected from this woylie on this occasion) microscopy was used alone to confirm the morphological presence of two trypomastigotes on the blood slides.

In contrast, of the three woylies infected with T. copemani P2, two of them (WC2807 and WC2930) lost the blood form of the parasite to below PCR detectable levels in the full $300 \mu \mathrm{l}$ of blood from March and April 2012 (Table 2). These negative PCR results were supported by the absence of trypomastigotes in the thin blood smears when examined by microscopy. In an effort to strengthen the reliability of these negative results from March and April 2012, nine additional nested PCR reactions were performed on each of the DNA extractions from WC2807 and WC2930. All 40 nested PCR reactions returned a negative result. The November 2011 and April 2012 positive samples were sequenced and confirmed the continued presence of T. copemani $\mathrm{P} 2$.

\section{Trypanosoma vegrandis sp. nov. - microscopy and image} acquisition

During sampling at KWS in February 2012, wet blood mounts were made from four woylies previously sampled in September 2011 and shown by PCR to be mono-infected with $T$. vegrandis sp. nov. The movement

Table 1 Morphological traits of the trypomastigotes of T.copemani: mean measurements ( $\mu \mathrm{m}) \pm$ standard error (range)

\begin{tabular}{|c|c|c|c|}
\hline $\begin{array}{l}\text { Measurements } \\
(\mu \mathrm{m})\end{array}$ & $\begin{array}{l}\text { Overall } \\
(\mathrm{N}=110)\end{array}$ & $\begin{array}{c}\mathrm{P} 1 \\
(\mathrm{~N}=82)\end{array}$ & $\begin{array}{c}\mathrm{P} 2 \\
(\mathrm{~N}=28)\end{array}$ \\
\hline L & $37.34 \pm 0.33(30.25-45.19)$ & $36.35 \pm 0.35(30.25-45.19)$ & $40.26 \pm 0.53(33.41-43.84)$ \\
\hline W & $6.12 \pm 0.18(1.15-10.23)$ & $5.98 \pm 0.21(1.51-10.23)$ & $6.53 \pm 0.31(3.71-9.24)$ \\
\hline PK & $11.44 \pm 0.22(3.93-15.89)$ & $11.49 \pm 0.27(3.93-15.89)$ & $11.27 \pm 0.35(5.43-14.79)$ \\
\hline PN & $15.42 \pm 0.23(7.34-19.53)$ & $14.98 \pm 0.27(7.34-19.04)$ & $16.71 \pm 0.32(11.10-19.53)$ \\
\hline $\mathrm{KN}$ & $4.36 \pm 0.10(2.52-7.31)$ & $3.92 \pm 0.07(2.52-5.80)$ & $5.66 \pm 0.17(4.19-7.31)$ \\
\hline NA & $15.85 \pm 0.23(9.30-22.06)$ & $15.44 \pm 0.26(9.30-22.06)$ & $17.06 \pm 0.36(13.15-20.91)$ \\
\hline $\mathrm{NI}$ & $0.98 \pm 0.02(0.39-1.42)$ & $0.98 \pm 0.02(0.39-1.42)$ & $0.99 \pm 0.03(0.60-1.37)$ \\
\hline $\mathrm{Kl}$ & $3.64 \pm 0.08(1.38-5.96)$ & $3.85 \pm 0.09(1.38-5.96)$ & $3.02 \pm 0.10(1.96-4.20)$ \\
\hline $\mathrm{FF}$ & $8.24 \pm 0.19(3.44-12.39)$ & $8.17 \pm 0.22(3.44-12.39)$ & $8.44 \pm 0.32(4.38-12.35)$ \\
\hline $\mathrm{K}-\mathrm{I}$ & $1.02 \pm 0.02(0.61-1.31)$ & $1.06 \pm 0.02(0.76-1.31)$ & $0.92 \pm 0.04(0.61-1.25)$ \\
\hline K-W & $0.71 \pm 0.01(0.50-1.00)$ & $0.72 \pm 0.01(0.50-1.00)$ & $0.69 \pm 0.02(0.50-0.93)$ \\
\hline
\end{tabular}




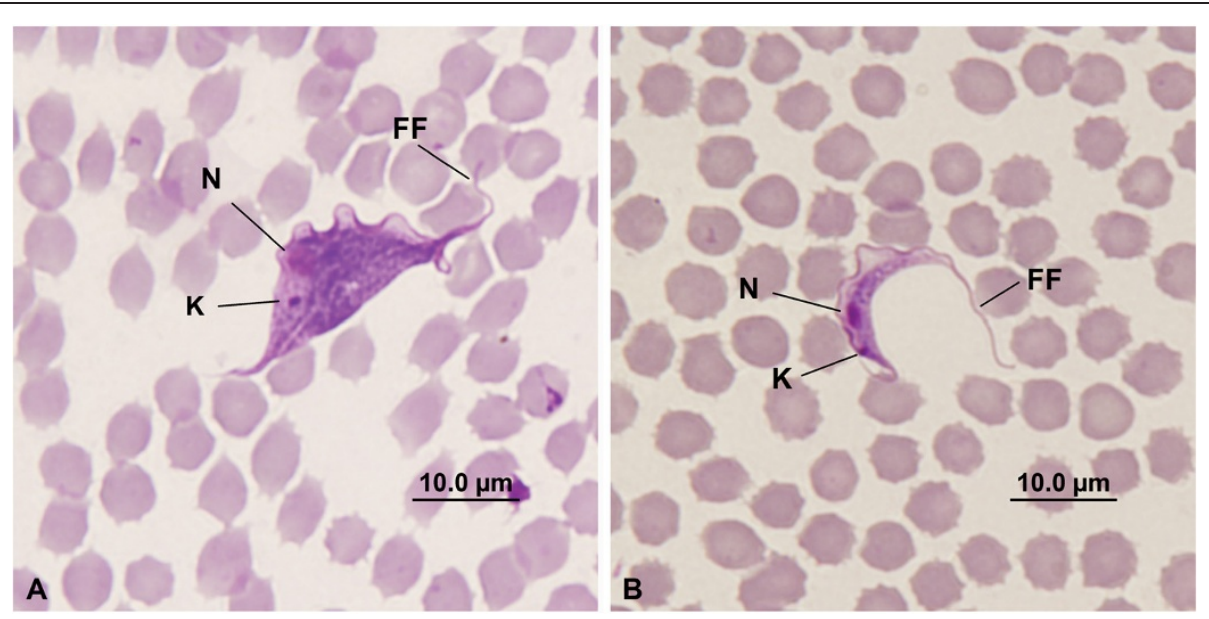

Figure 2 The different morphological forms of T. copemani identified from the same host. A - Broad trypomastigote form. B - Slender trypomastigote form. $\mathrm{K}=$ Kinetoplast, $\mathrm{N}=$ Nucleus, $\mathrm{FF}=$ Free Flagellum.

of this novel motile flagellated trypanosome was observed by microscopy as being a characteristic "corkscrew type" action.

A total of 20 trypanosomes were identified in stained blood smears from these same four woylies (Woylie ID: 7199222, 7236356, 7225370 \& K734) and measured. All were identified as trypomastigote forms (Figure 4). The overall measurements of these 20 trypomastigotes are presented in Table 3.

When considering the phylogenetic analysis presented by Botero et al., [19] and the sequencing PCR results below, the 20 trypomastigotes measured were separated into two groups. The first group was " $T$. vegrandis sp. nov. Genotype 1" (G1), comprising trypomastigotes found from Woylie ID: 7199222 \& 7236356 and the second was " $T$. vegrandis sp. nov. Genotype 2" (G2) comprising trypomastigotes found in Woylie ID: 7225370 \& K734. Mean morphological traits for each group are shown in Table 3. There were no significant differences between groups using all morphological traits in MANOVA $\left(\mathrm{F}_{7,12}=\right.$ $0.97, \mathrm{P}=0.49$ ) or using each trait separately in univariate ANOVA's. This genetically varied clade appears to be represented by a single, morphologically unique phenotype (Figure 4).

During the April 2012 sampling at NAR, blood mounts from one woylie known to be PCR positive for infections with $T$. vegrandis sp. nov. (WC2741; Table 4) were successfully hybridised and stained. Three trypomastigotes were identified by the hybridisation of the fluorescence probe in situ (Figure 5). The morphological measurements of these three trypomastigotes are presented in Table 3.

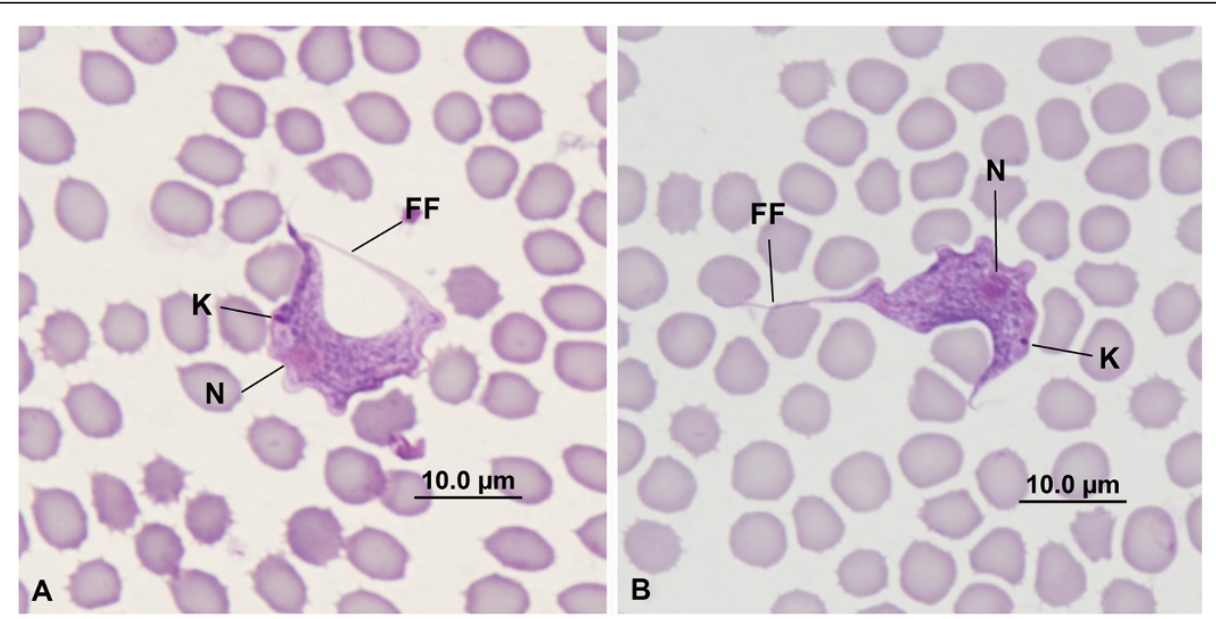

Figure 3 The different morphological phenotypes of $\boldsymbol{T}$. copemani identified from different hosts. A - T. copemani Phenotype 1 (P1). B - T. copemani Phenotype 2 (P2). K= Kinetoplast, $\mathrm{N}=$ Nucleus, FF= Free Flagellum. 
Table 2 PCR results showing the presence of T. copemani P1 and P2 tested at NAR between April 2011 and April 2012

\begin{tabular}{|c|c|c|c|c|c|c|c|c|}
\hline Woylie ID & Trypanosome & April & June & July & Nov & Dec & March & April \\
\hline number & species & 2011 & 2011 & 2011 & 2011 & 2011 & 2012 & 2012 \\
\hline WC2741 & T. copemani P1 & + & + & + & + & + & + & + \\
\hline WC2830 & T. copemani P1 & + & + & + & + & + & + & + \\
\hline WC2842 & T. copemani P1 & + & + & + & + & $-*$ & & + \\
\hline WC2844 & T. copemani P1 & & + & + & + & + & + & + \\
\hline WC2920 & T. copemani P1 & + & + & + & + & + & + & + \\
\hline WC2807 & T. copemani P2 & + & + & - & + & + & - & - \\
\hline WC2841 & T. copemani P2 & + & + & + & + & + & + & + \\
\hline WC2930 & T. copemani P2 & + & + & + & + & + & - & - \\
\hline
\end{tabular}

[+] PCR positive for T. copemani.

[-] PCR negative for T. copemani.

[] Woylie not trapped that month.

[*] PCR negative but positive to T. copemani by microscopy.

Trypanosoma vegrandis sp. nov. - PCR and sequencing

The clade-specific nested PCR confirmed the presence of T. vegrandis sp. nov. in four woylies at KWS (Woylie ID: 7199222, 7236356, 7225370 \& K734) during the trapping sessions of September 2011 and February 2012. These same four woylies tested negative to T. copemani and T. gilletti. Two distinct genotypes were identified by the sequencing of the PCR products, the first group was " $T$. vegrandis sp. nov. G1", again comprising trypomastigotes found in Woylie ID: 7199222 \& 7236356 (representing G3 and G6 genetic sequences of Clade B [19]). The second group was "T. vegrandis sp. nov. G2", again comprising trypomastigotes found in Woylie ID: 7225370 \& K734 (representing G4, G5 and G7 genetic sequences of Clade B [19]).

Of the five $T$. copemani $\mathrm{P} 1$ positive woylies at NAR, three of them (WC2741, WC2844 and WC2920) were also PCR positive for T. vegrandis sp. nov. Both WC2741 and WC2844 maintained a consistent mixed infection during the 13 month sampling period (Table 4). Of the three woylies infected with $T$. copemani P2, there was only one occasion that $T$. vegrandis sp. nov. was detected by the species-specific PCR as a mixed infection within the blood; this being WC2841 in December 2011 (Table 4).

All attempts to amplify $T$. vegrandis sp. nov. in the mixed presence of T. copemani using the sequencing PCR protocol failed. Also all attempts to amplify $T$. vegrandis sp. nov. with the T. gilletti species-specific PCR protocol failed.

\section{Description of a new species- Trypanosoma vegrandis} sp. nov.

Based on results presented here we propose the name Trypanosoma vegrandis sp. nov. for this morphologically and genetically distinct species found within the woylie.

Diagnosis: Morphological analysis of various blood forms from the woylie or Brush-tailed Bettong (B. penicillata) including microscopy of live motile bodies, fluorescence in situ hybridisation and Modified Wright's staining of fixed trypomastigotes. Description represents a single phenotype
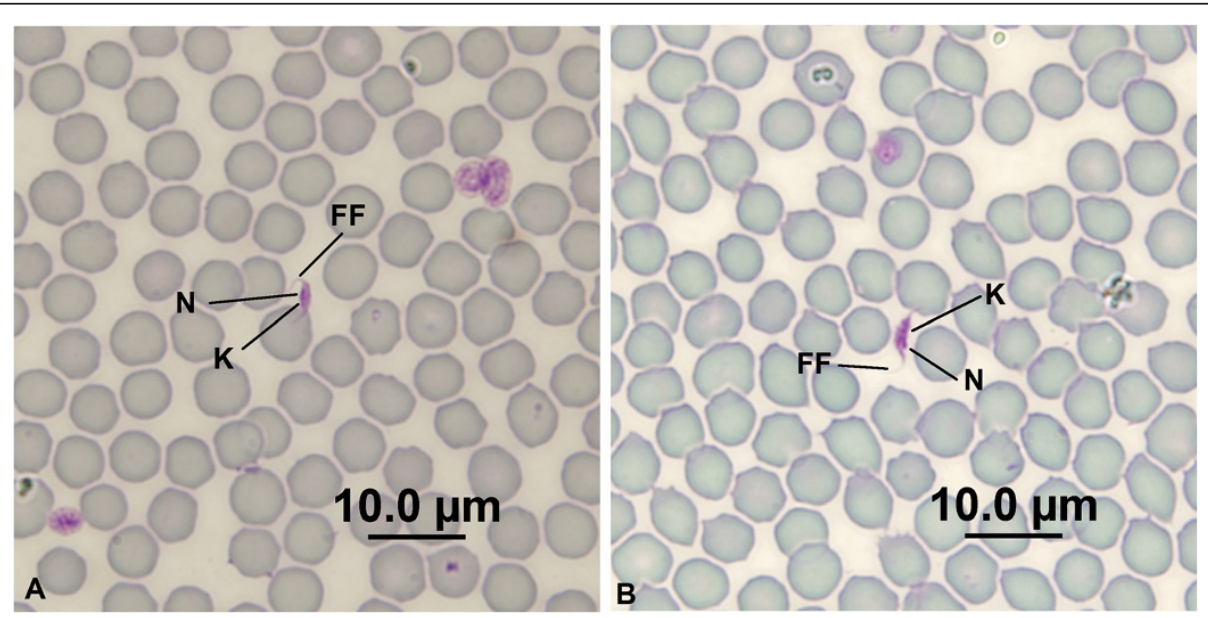

Figure 4 The morphological form of $\boldsymbol{T}$. vegrandis sp. nov. A and $\mathbf{B}$ - Trypomastigote forms. K= Kinetoplast, N= Nucleus, FF= Free Flagellum. 
Table 3 Morphological traits of the trypomastigotes of $T$. vegrandis sp. nov.: mean measurements $(\mu \mathrm{m}) \pm \mathrm{standard}$ error (range)

\begin{tabular}{|c|c|c|c|c|}
\hline $\begin{array}{l}\text { Measurements } \\
\qquad(\mu \mathrm{m})\end{array}$ & $\begin{array}{l}\text { Overall } \\
(\mathrm{N}=20)\end{array}$ & $\begin{array}{c}\mathrm{G} 1 \\
(\mathrm{~N}=10)\end{array}$ & $\begin{array}{c}\mathrm{G} 2 \\
(\mathrm{~N}=10)\end{array}$ & $\begin{array}{c}\text { Genotype unknown } \\
\text { FISH (N=3) }\end{array}$ \\
\hline L & $8.30 \pm 0.28(6.92-10.50)$ & $8.12 \pm 0.31(6.92-10.25)$ & $8.47 \pm 0.47(7.02-10.50)$ & $8.85^{*}$ \\
\hline W & $1.33 \pm 0.04(1.00-1.63)$ & $1.33 \pm 0.06(1.04-1.63)$ & $1.33 \pm 0.05(1.00-1.57)$ & $1.37 \pm 0.03(1.32-1.41)$ \\
\hline PK & $3.26 \pm 0.09(2.71-3.87)$ & $3.30 \pm 0.12(2.78-3.87)$ & $3.22 \pm 0.14(2.71-3.87)$ & $2.95 \pm 0.12(2.81-3.18)$ \\
\hline PN & $4.39 \pm 0.15(3.28-5.68)$ & $4.28 \pm 0.15(3.28-4.78)$ & $4.49 \pm 0.26(3.46-5.68)$ & $4.19 \pm 0.16(3.96-4.49)$ \\
\hline $\mathrm{KN}$ & $1.22 \pm 0.07(0.85-1.95)$ & $1.11 \pm 0.05(0.95-1.39)$ & $1.34 \pm 0.12(0.85-1.95)$ & $1.24 \pm 0.05(1.15-1.31)$ \\
\hline NA & $2.16 \pm 0.11(1.56-3.27)$ & $2.02 \pm 0.12(1.56-2.87)$ & $2.30 \pm 0.17(1.76-3.27)$ & $2.62 \pm 0.10(2.47-2.82)$ \\
\hline $\mathrm{NI}$ & $2.10 \pm 0.09(1.42-2.80)$ & $2.18 \pm 0.13(1.59-2.80)$ & $2.01 \pm 0.13(1.42-2.61)$ & $1.61 \pm 0.11(1.46-1.82)$ \\
\hline $\mathrm{KI}$ & $3.70 \pm 0.15(2.70-4.95)$ & $3.92 \pm 0.21(3.24-4.94)$ & $3.47 \pm 0.19(2.70-4.95)$ & $3.37 \pm 0.06(3.25-3.44)$ \\
\hline $\mathrm{FF}$ & $1.86 \pm 0.10(1.24-2.88)$ & $1.83 \pm 0.17(1.28-2.88)$ & $1.89 \pm 0.13(1.24-2.57)$ & $2.40^{*}$ \\
\hline
\end{tabular}

* Sample Number $=1$.

encompassing two different genotypes identified by phylogenetic analysis of the $18 \mathrm{~S}$ rDNA and gGAPDH gene [19].

The trypomastigotes of $T$. vegrandis sp. nov. have a curved body which is drawn out to a pointed posterior end. They are a small trypanosome with a smallest recorded length being $6.92 \mu \mathrm{m}$. The width of the trypanosome is about $16 \%$ that of its total length and the free flagellum is relatively long, being over $20 \%$ of the total length. The nucleus is located in the anterior half of the body, with the posterior kinetoplast positioned closer to the nucleus than to the posterior edge of the body. The distance between the nucleus and kinetoplast is about $20 \%$ of the body length, when excluding the free flagellum.

The mean total length of the trypomastigotes found in the blood of the woylie was $8.30 \mu \mathrm{m}$ (range: 6.92 $10.50 \mu \mathrm{m}$ ), mean width- $1.33 \mu \mathrm{m}$ (range: $1.00-1.63 \mu \mathrm{m}$ ), mean posterior edge to kinetoplast distance- $3.26 \mu \mathrm{m}$ (range: $2.71-3.87 \mu \mathrm{m}$ ), mean posterior edge to nucleus distance- $4.39 \mu \mathrm{m}$ (range: 3.28 - $5.68 \mu \mathrm{m}$ ), mean kinetoplast to nucleus distance- $1.22 \mu \mathrm{m}$ (range: $0.85-1.95 \mu \mathrm{m}$ ), mean nucleus to anterior edge distance- $2.16 \mu \mathrm{m}$ (range: 1.56 - $3.27 \mu \mathrm{m}$ ) and mean free flagellum length$1.86 \mu \mathrm{m}$ (range: $1.24-2.88 \mu \mathrm{m}$ ). The mean NI index was $2.10 \mu \mathrm{m}$ (range: $1.42-2.80 \mu \mathrm{m}$ ) and mean KI index was $3.70 \mu \mathrm{m}$ (range: $2.07-4.95 \mu \mathrm{m}$ ).

\section{Taxonomic summary}

Vertebrate type host Brush-tailed Bettong (Bettongia penicillata)

Vertebrate additional hosts Western Grey Kangaroo, Quenda, Tammar Wallaby, Chuditch [19]

\section{Invertebrate vector Unknown}

Morphological type location Karakamia Wildlife Sanctuary (S31.82073; E116.24604)

Table 4 PCR results showing the presence of T. vegrandis sp. nov. tested at NAR between April 2011 and April 2012

\begin{tabular}{|c|c|c|c|c|c|c|c|c|}
\hline $\begin{array}{l}\text { Woylie ID } \\
\text { number }\end{array}$ & $\begin{array}{l}\text { Trypanosome } \\
\text { species }\end{array}$ & $\begin{array}{l}\text { April } \\
2011\end{array}$ & $\begin{array}{l}\text { June } \\
2011\end{array}$ & $\begin{array}{l}\text { July } \\
2011\end{array}$ & $\begin{array}{l}\text { Nov } \\
2011\end{array}$ & $\begin{array}{c}\text { Dec } \\
2011\end{array}$ & $\begin{array}{c}\text { March } \\
2012\end{array}$ & $\begin{array}{l}\text { April } \\
2012\end{array}$ \\
\hline WC2741 & T. vegrandis sp. nov. & + & + & + & + & + & + & + \\
\hline WC2830 & T. vegrandis sp. nov. & - & - & - & - & - & - & - \\
\hline WC2842 & T. vegrandis sp. nov. & - & - & - & - & - & & - \\
\hline WC2844 & T. vegrandis sp. nov. & & + & + & + & + & + & + \\
\hline WC2920 & T. vegrandis sp. nov. & + & + & + & - & - & + & + \\
\hline WC2807 & T. vegrandis sp. nov. & - & - & - & - & - & - & - \\
\hline WC2841 & T. vegrandis sp. nov. & - & - & - & - & + & - & - \\
\hline WC2930 & T. vegrandis sp. nov. & - & - & - & - & - & - & - \\
\hline
\end{tabular}

[+] PCR positive for $T$. vegrandis sp. nov.

[-] PCR negative for $T$. vegrandis sp. nov.

[ ] Woylie not trapped that month. 

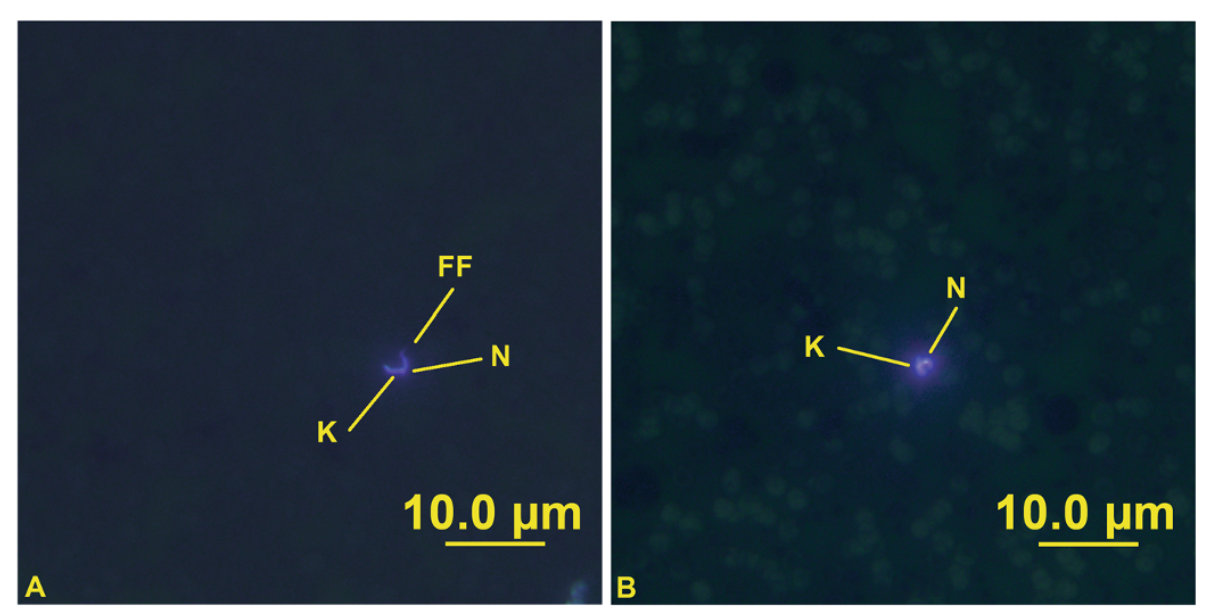

Figure 5 Fluorescence in situ hybridisation of $\boldsymbol{T}$. vegrandis sp. nov. A and $\mathbf{B}$ - Fluorescent trypomastigote forms. $\mathrm{K}=\mathrm{Kinetoplast,} \mathrm{N}=$ Nucleus, FF= Free Flagellum.

Additional locations Upper Warren Region (S34.11528; E116.32362), Native Animal Rescue (S31.86677; E115. 89072) and Dwellingup, WA

Site of infection Blood, brain (results not shown) as well as skeletal muscle, heart, lung, oesophagus, tongue, kidney, bone marrow, liver and spleen [19]

Pre-patent and patent periods Unknown

Subacute phase Unknown

\section{Chronic phase Unknown}

Etymology This species has been given the name vegrandis due to the small size; vegrandis is a logical name as it means diminutive, small and tiny.

\section{Mixed Infection- microscopy and image acquisition}

The mixed infection of $T$. copemani $P 1$ and $T$. vegrandis identified by molecular methodology for Woylie ID: WC2741 at NAR was confirmed by microscopy in April 2012 during the hybridization and staining procedure. When the fluorescent conditions of Figure $5 \mathrm{~B}$ were changed to white light microscopy, the field of view contained not only the hybridised $T$. vegrandis (Figure 6- circle) but also contained the thin trypomastigote of T. copemani P1 (Figure 6- arrow). Figures 4 and 6 also illustrate the varying translucency of the $T$. vegrandis trypomastigote forms when stained. The absence of fluorescence in the vicinity of $T$. copemani in Figure 5B confirmed the specificity of the probe.

\section{Discussion}

Overall, T. copemani trypomastigotes in the blood of woylies were characterised by a long curved body. The nucleus, on average, was near the centre of the body and the posterior, oval-shaped kinetoplast was positioned closer to the nucleus than to the posterior edge of the body. A free flagellum was present and was about $20 \%$ of the total length. The two phenotypes of T. copemani were distinguished by the statistically significant differences in a number of morphological traits, in particular the distance between the nucleus and kinetoplast. The KN distance for P1 was $3.92 \pm$ $0.07 \mu \mathrm{m}$ and for P2 was $5.66 \pm 0.17 \mu \mathrm{m}$. The different

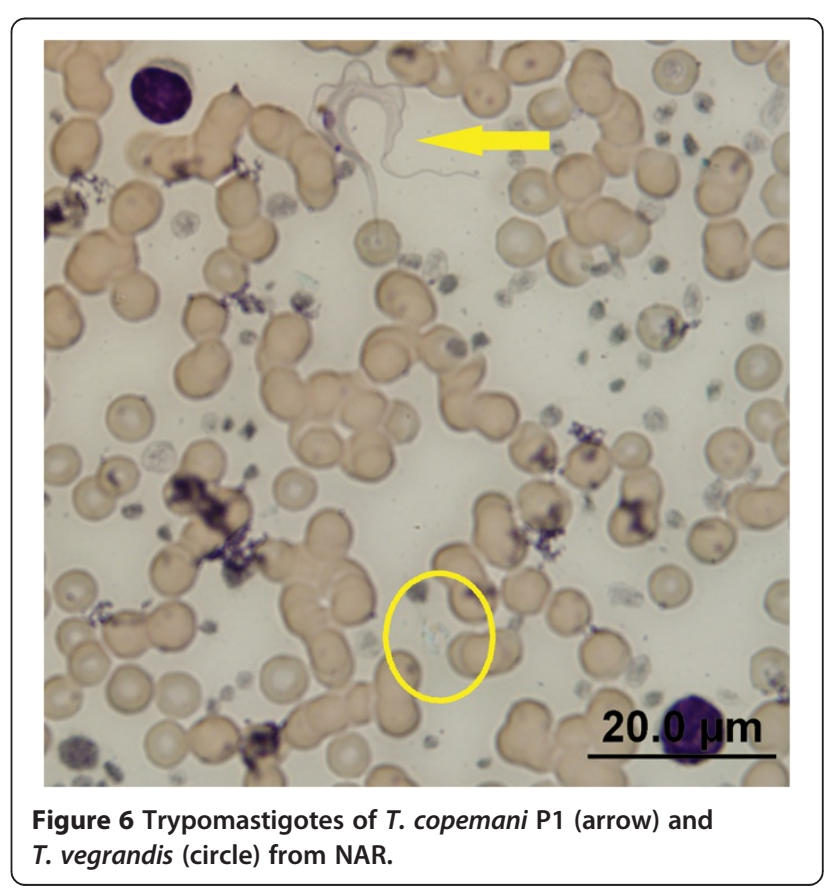


KN distances were not believed to be an artefact as all blood smears were made in exactly the same manner and the phenotypic grouping of the eight woylies at NAR matched exactly with the genotypic grouping of these same woylies.

From the T. copemani measurements, we suggest that the different thicknesses observed represent different life stages of the trypanosome within the host, as both broad and slender forms were observed simultaneously within individual P1 and P2 infected hosts. We believe that the broad trypomastigote was the blood form responsible for the reproductive phase (71\% of trypomastigotes measured) and the slender form was the adult trypomastigote. Similar size variations of the trypomastigote blood forms have been observed in various mammalian hosts and includes T. lewisi, T. musculi, T. evotomys and T. zapi [21]. Varying trypomastigote thickness was also observed in the Gilbert's Potoroo (Potorous gilbertii), with $T$. copemani trypomastigotes grouped as slender, medium and broad [24].

There appears to be host-induced morphological variation of trypanosomes found infecting wildlife. The morphology of $T$. copemani observed in the woylie, for example, differs to that of T. copemani found in Gilbert's potoroo, being relatively longer and thinner. The smallest length recorded in the woylie was $30.25 \mu \mathrm{m}$, while in the potoroo it was $25.0 \mu \mathrm{m}$ and the widest length recorded in the woylie was $10.23 \mu \mathrm{m}$, while in the potoroo it was $15.4 \mu \mathrm{m}$ [24]. Other dissimilarities included a larger PK mean in the woylie $(11.44 \mu \mathrm{m}$ compared to $8.1 \mu \mathrm{m}$ in the potoroo), a smaller $\mathrm{KN}$ and $\mathrm{FF}$ mean in the woylie $(4.36 \mu \mathrm{m}$ and $8.24 \mu \mathrm{m}$ compared to $5.8 \mu \mathrm{m}$ and $10.8 \mu \mathrm{m}$ respectively in the potoroo) [24]. Also dividing trypomastigotes of $T$. copemani were identified in the potoroo [24], whereas we failed to locate any divisional forms in the woylies. Compared to T. copemani in the quokka (Setonix brachyurus), trypomastigotes of T. copemani in the woylie were wider $(6.16 \mu \mathrm{m}$ compared to $4.2 \mu \mathrm{m}$ in the quokka), had a larger PK and NA mean (11.44 $\mu \mathrm{m}$ and $15.85 \mu \mathrm{m}$ compared to $6.5 \mu \mathrm{m}$ and $13.7 \mu \mathrm{m}$ respectively in the quokka) and a smaller $\mathrm{KN}$ and FF mean (4.36 $\mu \mathrm{m}$ and $8.24 \mu \mathrm{m}$ compared to $5.9 \mu \mathrm{m}$ and $12.1 \mu \mathrm{m}$ respectively in the quokka) [24]. This polymorphism emphasises the importance of using both morphological and genetic criteria in describing trypanosomes from wildlife.

The two morphological phenotypes of T. copemani described in the present study, P1 and P2, correspond to Clade A Genotypes 1 and 2 respectively, of which only P2 ( $\approx$ G2) has the ability to invade and divide within the cells of the host [19]. Two woylies, which were sampled over time at NAR (WC2807 and WC2930), appeared to lose their infection with T. copemani P2; this was based on the lack of PCR detection of trypanosomes in the peripheral blood. This may be because the infection became localised to the tissues of the host. In contrast, woylies infected with $T$. copemani P1, including those concurrently infected with $T$. vegrandis consistently maintained detectable levels of $T$. copemani P1 during this study. Due to our small samples sizes for these observations, further work is required to determine if $T$. copemani P1 is capable of infecting tissues, which may indicate a significant difference in virulence potential between the two forms of T. copemani.

The chronic effect of $T$. copemani P2 within the woylie remains unknown. However, it has been hypothesised from histopathological observations that when T. copemani P2 invade host cells as part of the life cycle in the woylie, it may initiate a strong inflammatory response of the host, with significant tissue degeneration occurring in the heart, oesophagus, kidney and tongue [19]. The pathological lesions and tissue degeneration observed within infected woylies with $T$. copemani P2 show similarities to the pathological changes observed in infected opossums with T. cruzi $[19,25]$. Overtime these pathological changes to the woylies may reduce its fitness and be a contributing factor during its recent decline.

The naming of $T$. vegrandis was supported by microscopic visualisation of live motile stages, Modified Wright's stained trypomastigotes and hybridised forms stained with a species-specific fluorescent probe. It is also complemented by the genetic amplification using speciesspecific Clade B primers, the failure of the $T$. vegrandis species-specific fluorescent probe to hybridise to T. copemani (Figure 5B \& 6), and failure of genetic amplification using the T. gilletti species-specific primers.

In spite of the genetic variability seen in the phylogenetic analysis of Clade B by Botero et al., [19] we observed morphological uniformity, grouping the genetic sequences of Clade B G3 - G7 together as a single morphological phenotype. This provides further support that the description of a trypanosome species should not be based on morphology alone, due to the polymorphic nature of the trypomastigotes in the blood (as discussed above with $T$. copemani in different hosts). As such, further studies are required to determine whether $T$. gilletti has morphological affinities with $T$. vegrandis, since $T$. gilletti was described solely on genetic data [26].

The morphology of $T$. vegrandis has been elusive since its molecular identification and in hindsight, is not surprising that it was not detected in previous studies when its small size and translucent nature are taken into account. We now know that $T$. vegrandis is the most prevalent trypanosome within this sub-population of woylies at KWS (unpublished data) but may well have been overlooked in previous surveys at KWS [9] and other locations [8,27], because of the limitations of the molecular tools used at the time. 
Another reason for the absence of previous morphological observations may be the critical timing between blood collection and fixation of the slide. We believe that the slides need to be fixed and stained within the 24 hour period after blood collection. Increasing the time for fixation of the blood slide results in a degradation of T. vegrandis, to a point where it is no longer detectable by microscopy (unpublished data). This was the case for samples collected at KWS in September 2011 where 22 PCR-positive blood smears were collected, and were fixed and stained four weeks later. By this time no morphological forms of $T$. vegrandis were identified by microscopy. It is therefore possible that this may have been the case for the molecular-based reports of $T$. gilletti from koalas and $T$. gilletti-like trypanosomes from woylies where no morphological forms were identified [26,27].

Overall, the trypomastigotes of $T$. vegrandis in the blood of woylies were approximately $20 \%$ the length of T. copemani, with a minimum length of $6.92 \mu \mathrm{m}$. The previous smallest reported individual trypomastigote lengths that we found were for $T$. congolense and $T$. simiae, both with a minimum length of $8 \mu \mathrm{m}$ [21]. T. vegrandis is believed to be one of the smallest trypanosomes reported infecting mammals [21,24,26,28-37]. Using the smallest length of both $T$. vegrandis and $T$. copemani from this study and the comprehensive analysis of trypanosome species complied by Hoare [21], Figure 7 compares the smallest individual recorded length for each sub-genus, as well as the mean of the smallest recorded lengths of the species within each sub-genus $(\mathrm{N}=104)$.

T. copemani was grouped within the subgenus Herpetosoma due to the long free flagellum, oval kinetoplast, and the relatively large distance between the kinetoplast and nucleus [24]. Surprisingly, similar morphological ratios of $T$. vegrandis are reported here. Apart from the very small size of $T$. vegrandis, all of the body proportions (except for the NI index) are similar to that of $T$. copemani, with a relatively long free flagellum and a relatively large distance between the kinetoplast and nucleus. At this stage it is very difficult to assign $T$. vegrandis to a subgenus. The phylogenetic analysis of the $18 \mathrm{~S}$ rDNA and $g G A P D H$ genes suggest that $T$. vegrandis may be part of the Stercoraria grouping of trypanosomes as it shares a close evolutionary relationship with $T$. copemani and $T$. pestanai (both being part of the Stercoraria group) [19,21]. Further work is required to understand the transmission dynamics from vector to host, along with the life cycle of the trypanosome in the vector before this can be commented on further.

\section{Conclusion}

In this report we describe the morphological polymorphism of $T$. copemani, which includes the different trypomastigote phenotypes from the blood of woylies. We also provide the first morphological observations and taxonomic description of trypanosomes from a new genetically diverse clade, for which we propose the name $T$. vegrandis. Up until now this small trypanosome has only been identified by PCR from a variety of hosts, including the woylie. Using fluorescence in situ hybridisation and light microscopy, we described a mixed trypanosome infection in a woylie, with both $T$. copemani and $T$. vegrandis observed. The temporal reduction of $T$. copemani P2 in the peripheral blood of the woylie and its ability to invade cells may

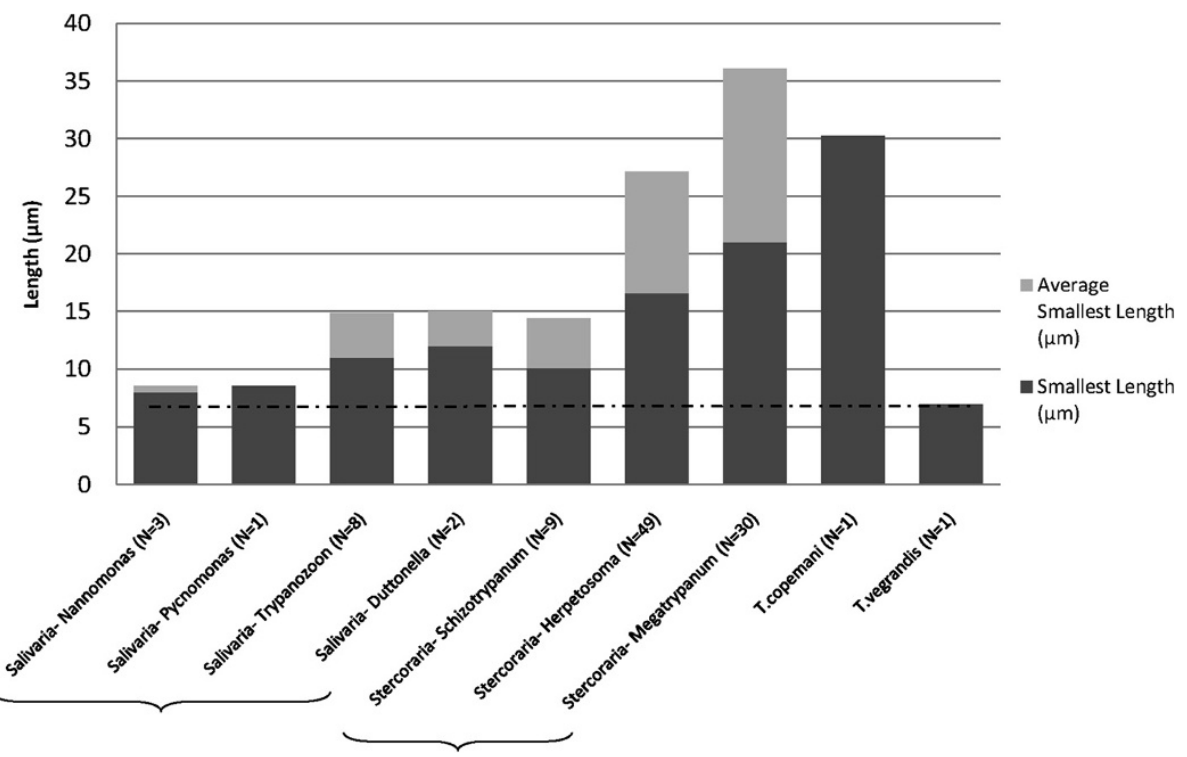

Figure 7 Smallest reported length and average smallest length of the species within each subgenus of Trypanosoma [21], T. copemani and $T$. vergrandis. 
suggest that this more virulent phenotype could become localised within the tissues of the host. Over time, tissue degeneration of the host could result in an overall reduced fitness, making the woylie more susceptible to predation in the wild.

\section{Competing interests}

The author(s) declare that they have no competing interests.

\section{Authors' contributions}

CKT, AB, AFW and RCAT designed the study; CKT, AFW, SSG, AJL and RCAT implemented the study; CKT managed the data; CKT, AB, SSG and AJL analysed and interpreted the data; CKT wrote the paper. CKT, AFW, SSG and RCAT supervised the different phases of the study. All authors read, revised and approved the final manuscript.

\section{Acknowledgements}

We would like to thank Manda Page, Jo Kuiper and Alexander Kabat at KWS, Marika Maxell, Chris Vellios and Colin Ward at DEC Science Division, Manjimup, Lizzie Aravidis and Kelli Ellemor at NAR, as well as all of the volunteers for their assistance in the collection of samples. We would also like to thank Andrew Li and Peter Adams for their assistance with developing the FISH protocol and Louise Pallant for her quidance with molecular techniques. Funding for this project was supplied to CKT by Murdoch University Australian Postgraduate Award and was supported by funding from the Western Australian Government's State NRM Program, to SSG by a Discovery Early Career Researcher Award (Australian Research Council). As a component of the collaborative, Woylie Conservation Research Project', lead by DEC, funding was also provided by DEC ('Saving Our Species' initiative), WA State NRM program and Caring for Our Country federal funds. Trapping of woylies at UWR was conducted by DEC, and at KWS by AWC and with animal ethics approval from the DEC AEC. The woylies were managed and handled using procedures formally approved by the Murdoch University Animal Ethics Committee (AEC number: W2350-10) and Department of Environment and Conservation Animal Ethics Committee (AEC number: DEC 36/2010) in compliance with the Australian Code of Practice for the use of Animals for Scientific Purposes. In accordance with section 8.5 of the ICZN's International Code of Zoological Nomenclature, details of the new species have been submitted to ZooBank with the life science identifier (LSID) zoobank. org:pub:3DA85A31-0B18-4BF6-A82D-079286D89A3F.

\section{Author details}

'School of Veterinary and Life Sciences, Murdoch University, South Street, Western Australia 6150, Australia. ${ }^{2}$ Science Division, Department of Environment and Conservation, Manjimup WA 6258, Australia. ${ }^{3}$ Fish Health Unit and Freshwater Fish Group, School of Veterinary and Life Sciences, Murdoch University, South Street, Western Australia 6150, Australia.

Received: 26 February 2013 Accepted: 22 April 2013

Published: 26 April 2013

\section{References}

1. Barrett MP, Burchmore RJS, Stich A, Lazzari JO, Frasch AC, Cazzulo JJ, Krishna S: The trypanosomiases. Lancet 2003, 362:1469-1480

2. Brun R, Hecker H, Lun ZR: Trypanosoma evansi and T. equiperdum: distribution, biology, treatment and phylogenetic relationship (a review). Vet Parasitol 1998, 79:95-107.

3. D'Archivio S, Medina M, Cosson A, Chamond N, Rotureau B, Minoprio P, Goyard S: Genetic engineering of Trypanosoma (Dutonella) vivax and in vitro differentiation under axenic conditions. PLOS Negl Trop Dis 2011, 5:e1461.

4. Steverding D: The history of African trypanosomiasis. Parasit Vectors 2008, 1:3.

5. Wyatt KB, Campos PF, Gilbert MTP, Kolokotronis SO, Hynes WH, DeSalle R, Daszak P, MacPhee RDE, Greenwood AD: Historical mammal extinction on Christmas Island (Indian Ocean) correlates with introduced infectious disease. PLoS One 2008, 3:1-9.

6. Andrews CW: An account of a visit to Christmas Island in 1908. J Zool 1987, 1909(1909):101-103.
7. Pickering J, Norris CA: New evidence concerning the extinction of the endemic murid Rattus macleari from Christmas Island, Indian Ocean. Aust Mammal 1996, 19:19-25.

8. Averis S, Thompson RCA, Lymbery AJ, Wayne AF, Morris KD, Smith A: The diversity, distribution and host-parasite associations of trypanosomes in Western Australian wildlife. Parasitology 2009, 136:1269-1279.

9. Smith A, Clark P, Averis S, Lymbery AJ, Wayne AF, Morris KD, Thompson RCA: Trypanosomes in a declining species of threatened Australian marsupial, the brush-tailed bettong Bettongia penicillata (Marsupialia: Potoroidae). Parasitology 2008, 135:1329-1335.

10. De Tores PJ, Start AN: Brush-tailed Bettong (Bettongia penicillata). In The Mammals of Australia. Edited by Van Dyck S, Strahan RJ. Sydney: Reed New Holland; 2008:291-292.

11. Wayne AF: Diagnosis of recent woylie (Bettongia penicillata ogilbyi) declines in southwestern Australia: Progress Report of the Woylie Conservation Research Project. Perth: Western Australia Government Department of Environment and Conservation; 2008.

12. Claridge AW, Seebeck JH, Rose R: Bettongs, Potoroos and the Musky Rat-Kangaroo. Melbourne: CSIRO Publishing; 2007.

13. Pacioni C, Wayne AF, Spencer PBS: Effects of habitat fragmentation on population structure and long distance gene flow in an endangered marsupial: the woylie. J Zool 1987, 283:98-107.

14. Abbott I: Historical perspectives of the ecology of some conspicuous vertebrate species in south-west Western Australia. Conserv Sci W Aust 2008, 6:1-214.

15. Bettongia penicillata ogilbyi in Species Profile and Threats Database. http:// www.environment.gov.au/cgi-bin/sprat/public/publicspecies.pl? taxon_id=66844.

16. Wayne AF, Maxwell M, Ward C, Vellios C, Ward B, Liddelow GL, Wilson I, Wayne JC, Williams MR: The importance of getting the numbers right: quantifying the rapid and substantial decline of an abundant marsupial, Bettongia penicillata. Wildl Res. in press.

17. Wayne A, Maxwell M, Nicholls P, Pacioni C, Reiss A, Smith A, Thompson RCA, Vellios C, Ward C, Wayne J, et al: The Woylie Conservation Research Project: investigating the cause(s) of woylie declines in the Upper Warren region. Perth: Western Australia Government Department of Environment and Conservation; 2011

18. Groom C: Justification for continued conservation efforts following the delisting of a threatened species: a case study of the woylie, Bettongia penicillata ogilbyi (Marsupialia : Potoroidae). Wildl Res 2010, 37:183-193.

19. Botero A, Thompson CK, Peacock C, Clode PL, Nicholls PK, Wayne AF, Lymbery AJ, Thompson RCA: Trypanosomes genetic diversity, polyparasitism and the population decline of the critically endangered Australian marsupial, the brush tailed bettong or woylie (Bettongia penicillata). Int J Parasitol Parasites Wildl 2013, 2:77-89.

20. Li A: Role of feral pig (Sus scrofa) in the dissemination of Phytophthora cinnamomi in South-western Australia. Murdoch University: PhD Thesis; 2012.

21. Hoare CA: The trypanosomes of mammals. Oxford, England: Blackwell Scientific Publishing; 1972.

22. JMP 10.0 SAS. Carey, NC: Institute Inc.

23. McInnes LM, Gillett A, Hanger J, Reid SA, Ryan UM: The potential impact of native Australian trypanosome infections on the health of koalas (Phascolarctos cinereus). Parasitology 2011, 138:1-11.

24. Austen JM, Jefferies R, Friend JA, Ryan U, Adams P, Reid SA: Morphological and molecular characterization of Trypanosoma copemani $\mathrm{n}$. sp. (Trypanosomatidae) isolated from Gilbert's potoroo (Potorous gilbertii) and quokka (Setonix brachyurus). Parasitology 2009, 136:783-792.

25. Teixeira ARL, Nascimento RJ, Sturm NR: Evolution and pathology in Chagas disease: a review. Mem Inst Oswaldo Cruz 2006, 101:463-491.

26. Mclnnes LM, Hanger J, Simmons G, Reid SA, Ryan UM: Novel trypanosome Trypanosoma gilletti sp. (Euglenozoa: Trypanosomatidae) and the extension of the host range of Trypanosoma copemani to include the koala (Phascolarctos cinereus). Parasitology 2011, 138:59-70.

27. Paparini A, Irwin PJ, Warren $K$, Mclnnes LM, De Tores $P$, Ryan UM: Identification of novel trypanosome genotypes in native Australian marsupials. Vet Parasitol 2011, 183:21-30.

28. McInnes LM, Gillett A, Ryan UM, Austen J, Campbell RS, Hanger J, Reid SA: Trypanosoma irwini n. sp (Sarcomastigophora: Trypanosomatidae) from the koala (Phascolarctos cinereus). Parasitology 2009, 136:875-885. 
29. Hamilton PB, Stevens JR, Holz P, Boag B, Cooke B, Gibson WC: The inadvertent introduction into Australia of Trypanosoma nabiasi, the trypanosome of the European rabbit (Oryctolagus cuniculus), and its potential for biocontrol. Mol Ecol 2005, 14:3167-3175.

30. Hatama S, Shibahara T, Suzuki M, Kadota K, Uchida I, Kanno T: Isolation of a Megatrypanum trypanosome from sika deer (Cervus nippon yesoensis) in Japan. Vet Parasitol 2007, 149:56-64.

31. Riedel D: Reproduction, Structure and Host Specificity of Trypanosoma (Herpetosoma) tamiasi sp. n. from the Eastern Chipmunk, Tamias striatus. J Eukaryot Microbiol 1975, 22:8-17.

32. Karbowiak G, Wita I: Trypanosoma (Herpetosoma) grosi kosewiense subsp. n., the parasite of the yellow-necked mouse Apodemus flavicollis (Melchior, 1834). Acta Protozool 2004, 43:173-178.

33. Sato H, Al-Adhami BH, Une Y, Kamiya H: Trypanosoma (Herpetosoma) kuseli sp. n. (Protozoa: Kinetoplastida) in Siberian flying squirrels (Pteromys volans). Parasitol Res 2007, 101:453-461.

34. Upton SJ, Fridell RA, Tilley M: Trypanosoma kansasensis sp. n. from Neotoma floridana in Kansas. J Wildl Dis 1989, 25:410-412.

35. Kingston N, Morton JK: Trypanosoma cervi sp. $\mathrm{n}$. from elk (Cervus canadensis) in Wyoming. J Parasitol 1975, 61:17-23.

36. Madeira MF, Sousa MA, Barros JHS, Figueiredo FB, Fagundes A, Schubach A, De Paula CC, Faissal BNS, Fonseca TS, Thoma HK: Trypanosoma caninum n. sp.(Protozoa: Kinetoplastida) isolated from intact skin of a domestic dog (Canis familiaris) captured in Rio de Janeiro, Brazil. Parasitology 2009, 136:411-423.

37. Bettiol SS, Jakes K, Le DD, Goldsmid JM, Hocking G: First Record of Trypanosomes in Tasmanian Bandicoots. J Parasitol 1998, 84:538-541.

doi:10.1186/1756-3305-6-121

Cite this article as: Thompson et al: Morphological polymorphism of Trypanosoma copemani and description of the genetically diverse $T$. vegrandis sp. nov. from the critically endangered Australian potoroid, the brush-tailed bettong (Bettongia penicillata (Gray, 1837)). Parasites \& Vectors 2013 6:121.

\section{Submit your next manuscript to BioMed Central and take full advantage of:}

- Convenient online submission

- Thorough peer review

- No space constraints or color figure charges

- Immediate publication on acceptance

- Inclusion in PubMed, CAS, Scopus and Google Scholar

- Research which is freely available for redistribution 